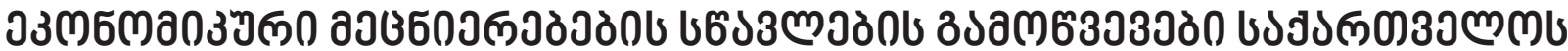

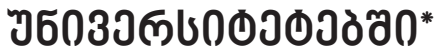

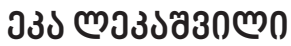

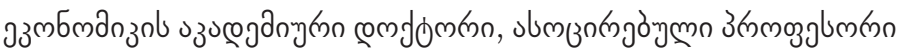

https://doi.org/10.35945/gb.2018.06.012

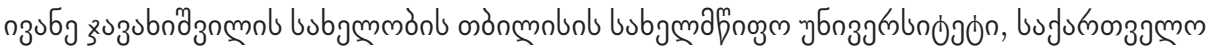

eka.lekashvili@tsu.ge

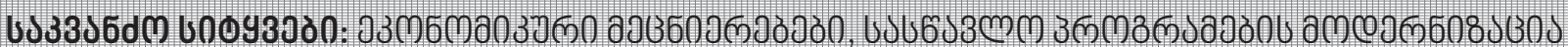

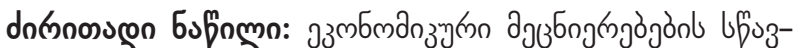

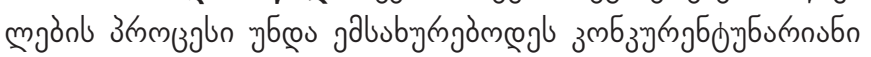

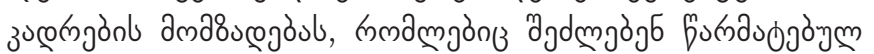

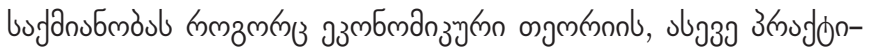

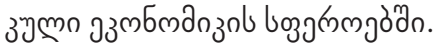

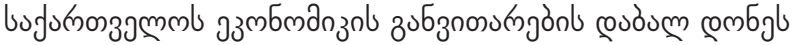

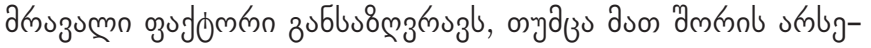

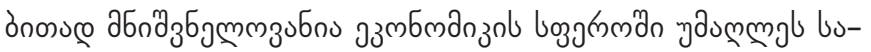

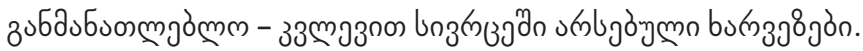

Бง

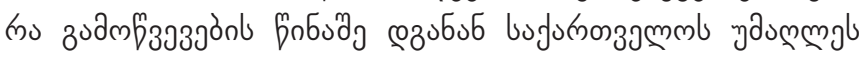

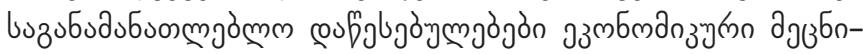

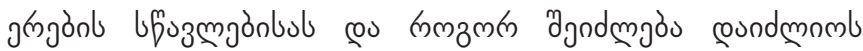

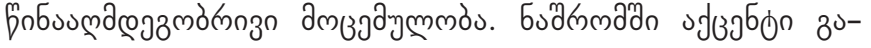

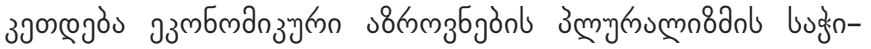

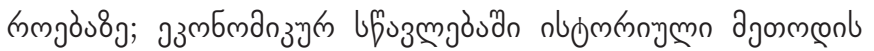

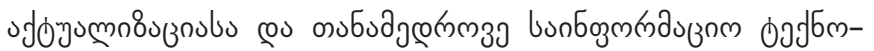

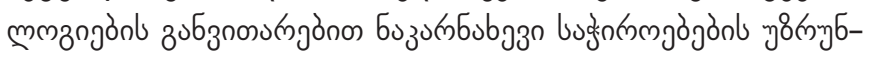

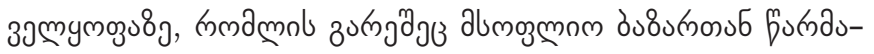

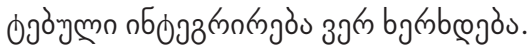

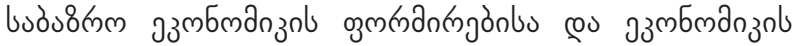
on

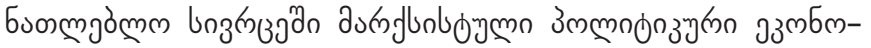

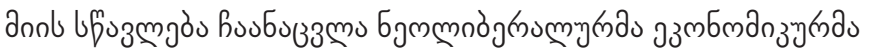

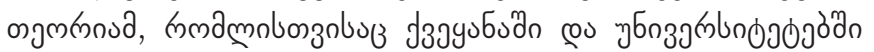

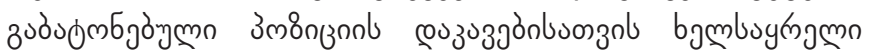

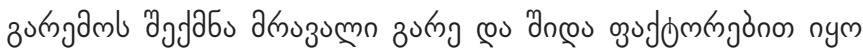

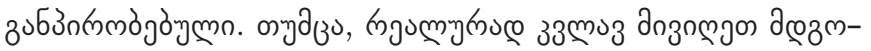

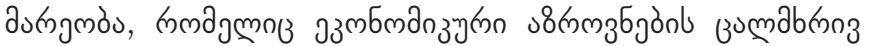

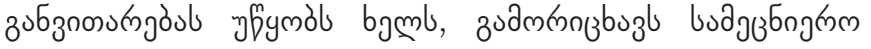

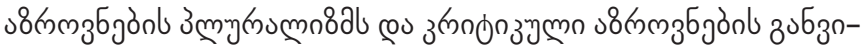
mungouls.

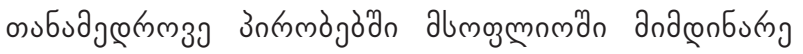

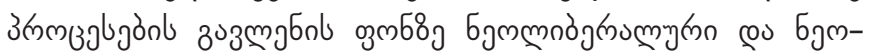

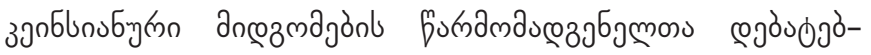

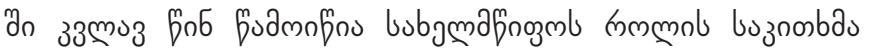

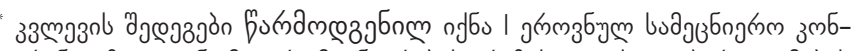

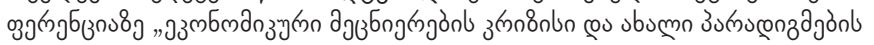
dngos"

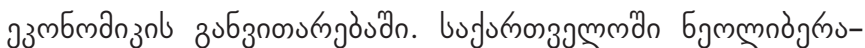

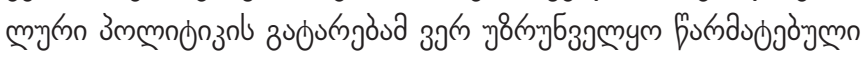

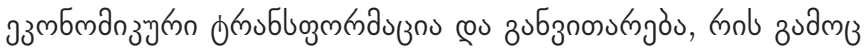

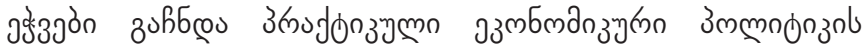

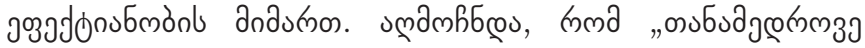

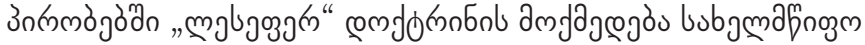

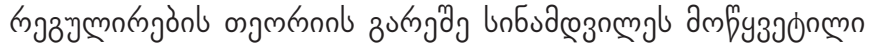

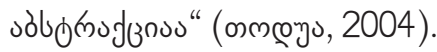

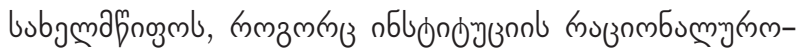

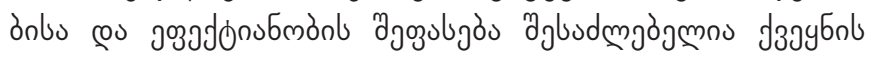

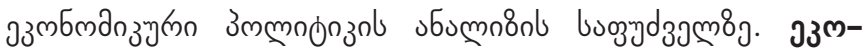

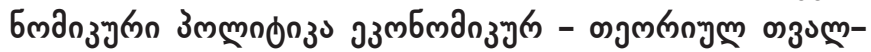

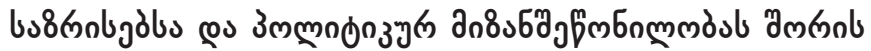

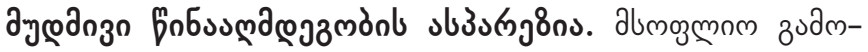

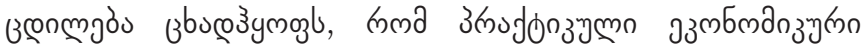

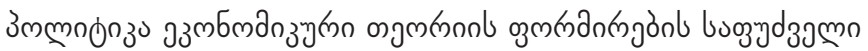

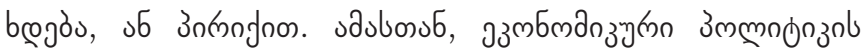

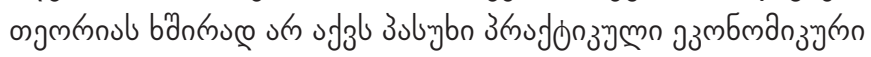

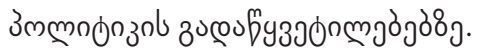

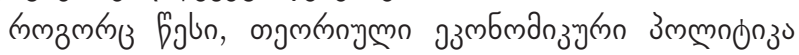

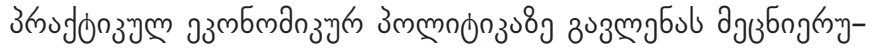

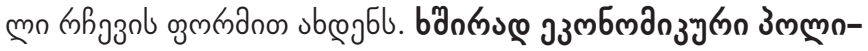

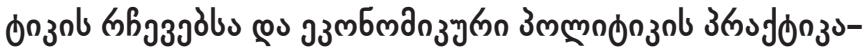

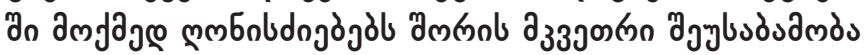

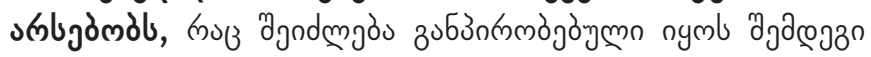

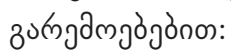

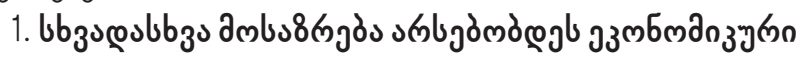

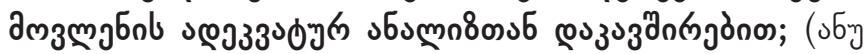

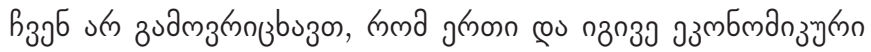

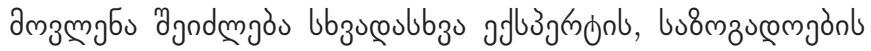

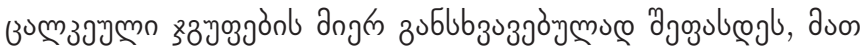

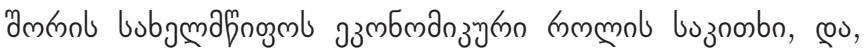

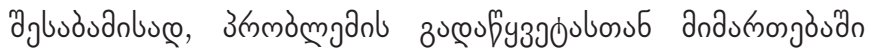

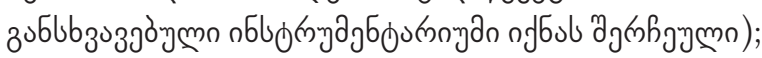

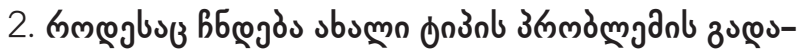

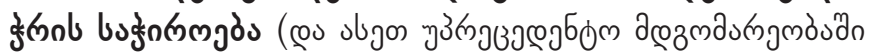

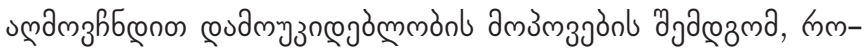

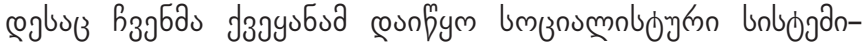

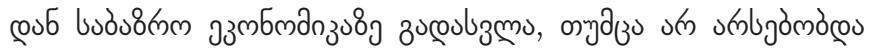




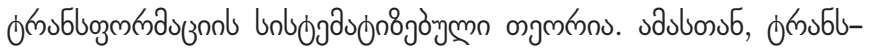

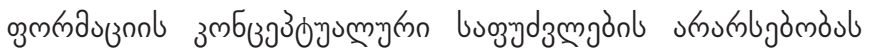

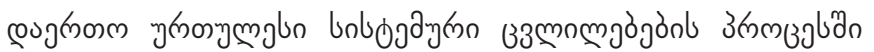

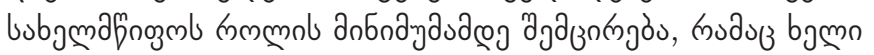

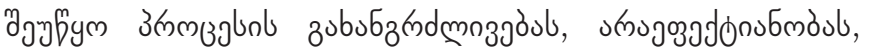

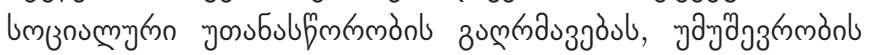

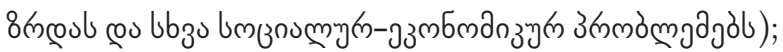

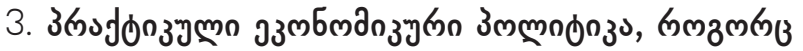

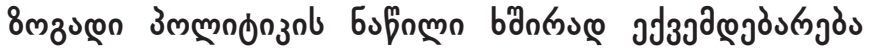

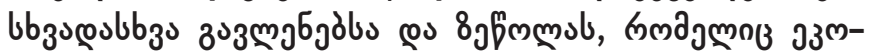

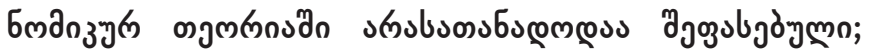

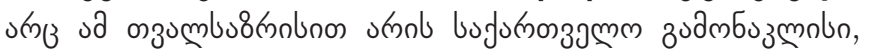

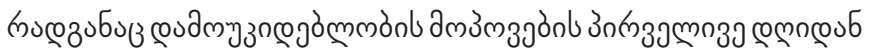

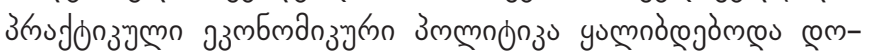

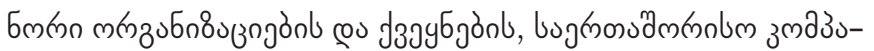

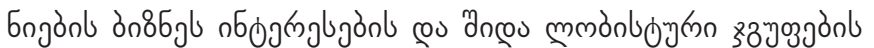

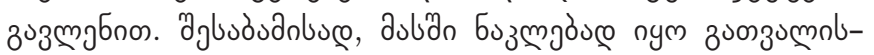
боб

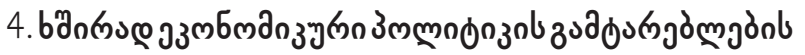

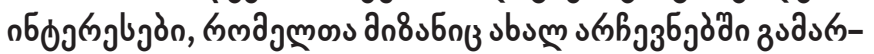

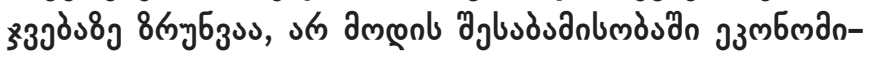

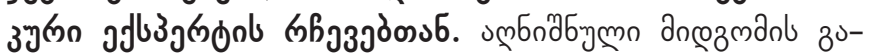

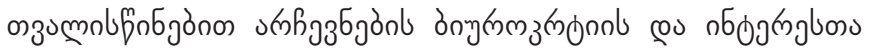

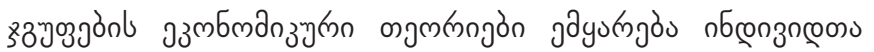

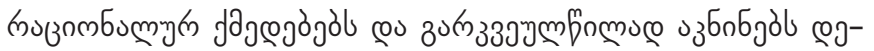

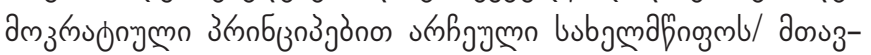

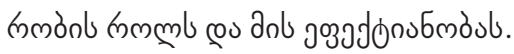

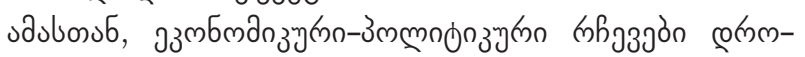

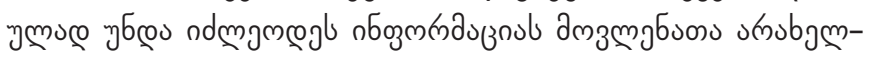

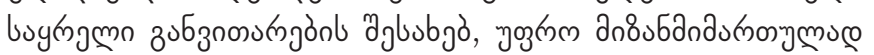

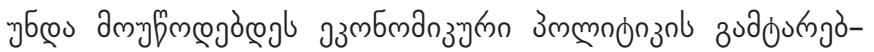

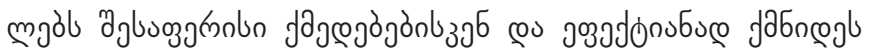

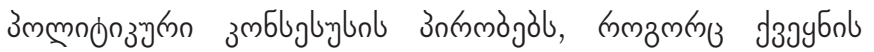

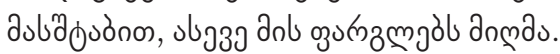

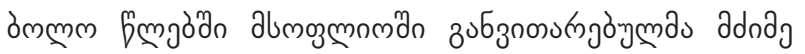

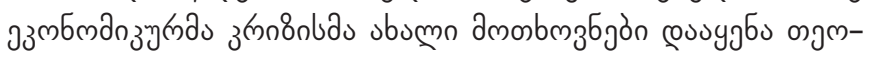

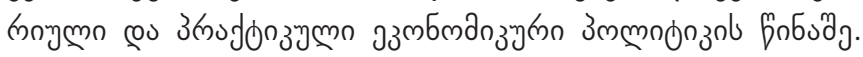

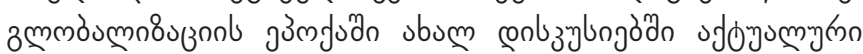

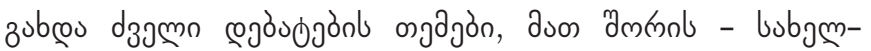

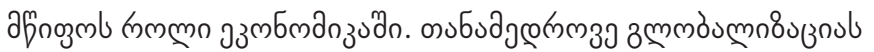

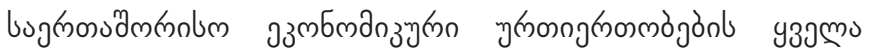

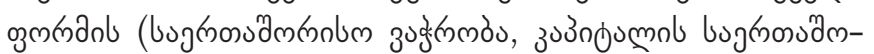

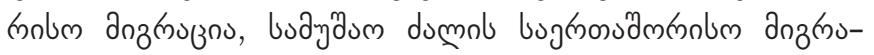

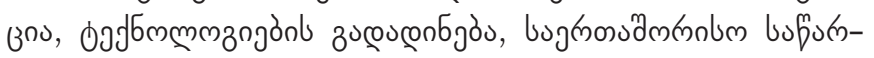

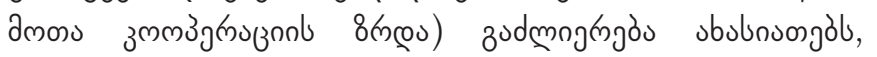

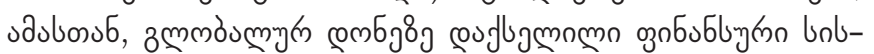

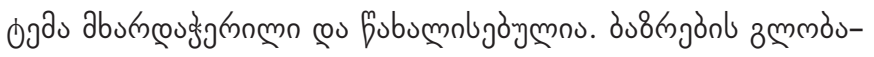

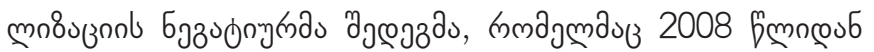
øुnбublym

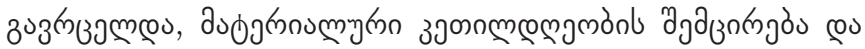

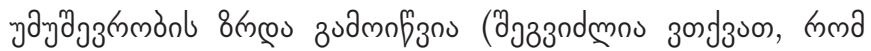

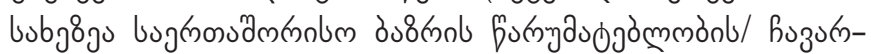
énol auzumnono).

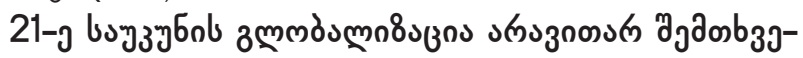

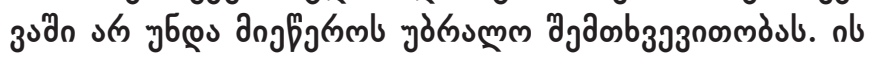

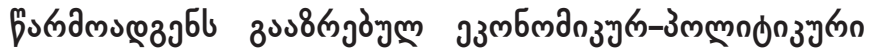

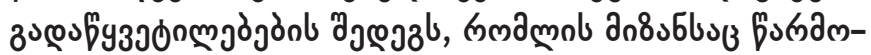

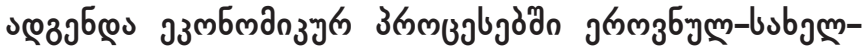

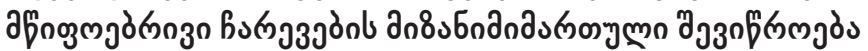

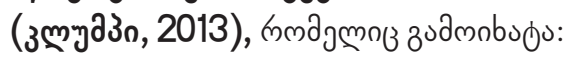

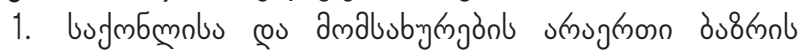

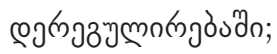

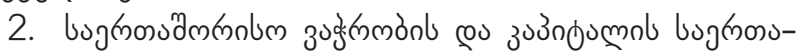

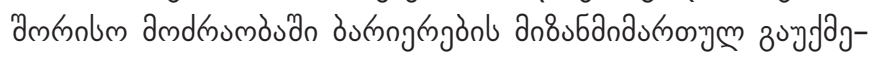
उodn;

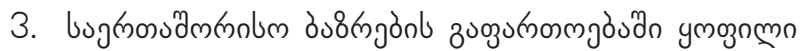

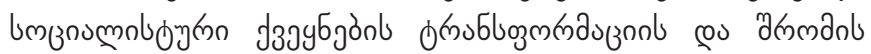

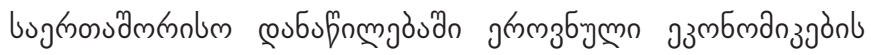

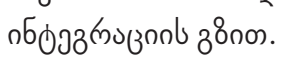

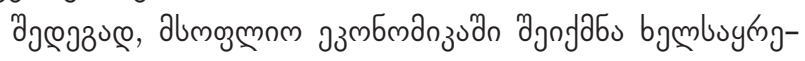

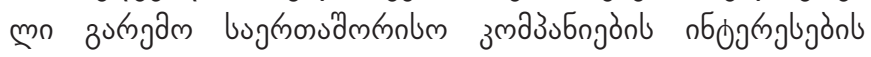
zuluojumadmus.

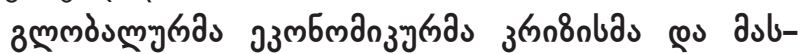

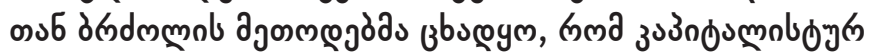

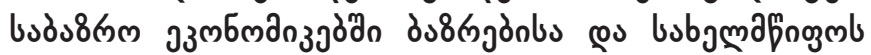

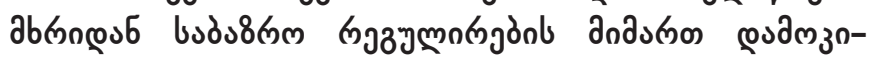

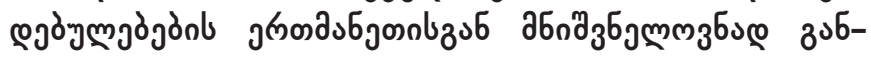

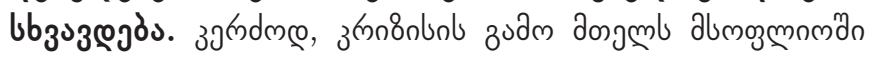

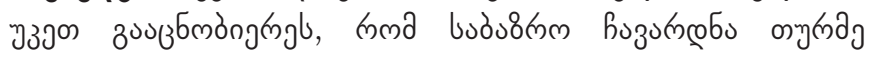

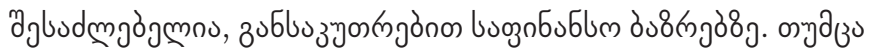

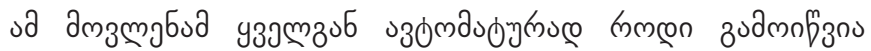

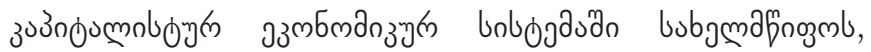

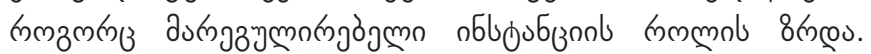

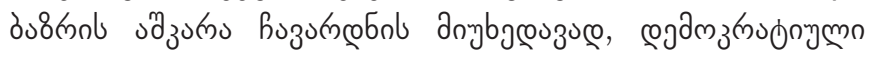

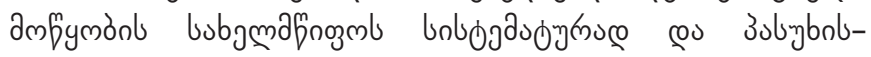

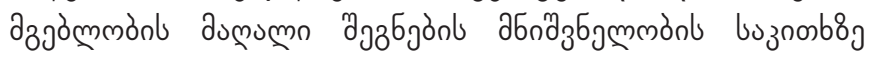

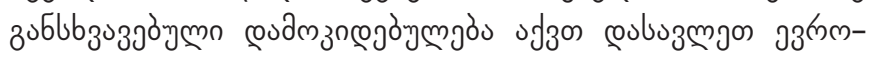

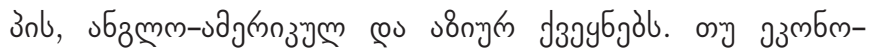

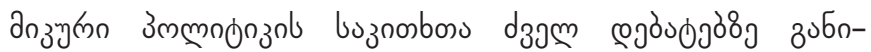

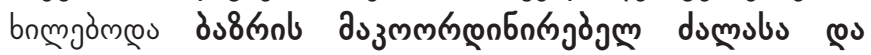

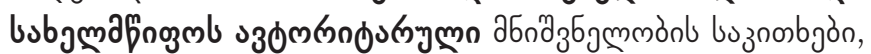

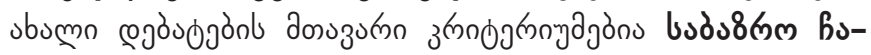

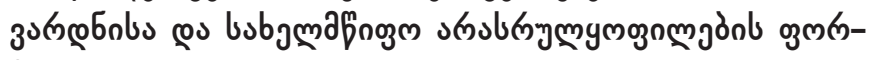

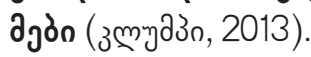

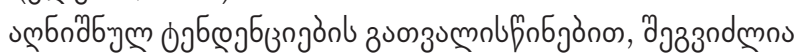

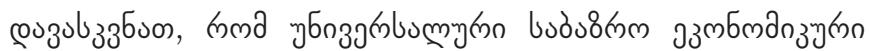
unboja s s u s

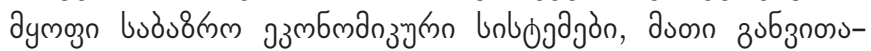




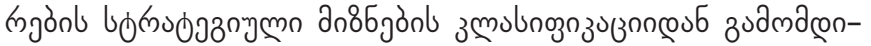

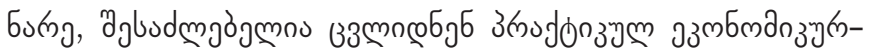

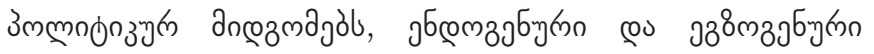

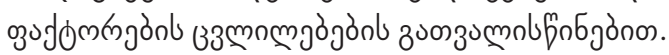

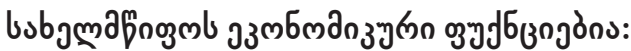

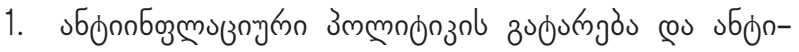

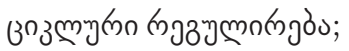

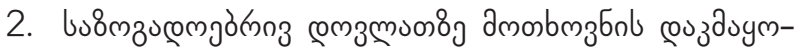

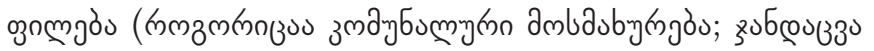

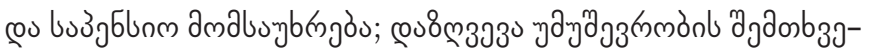

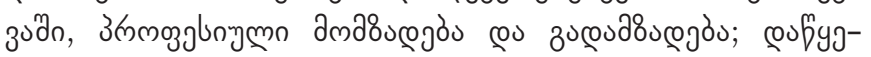

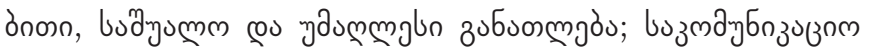

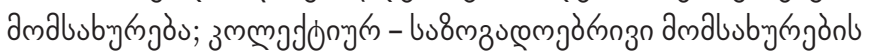

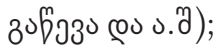

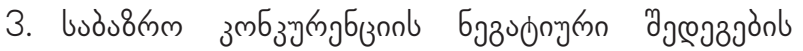

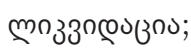

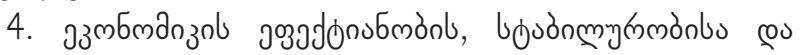

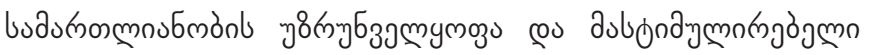

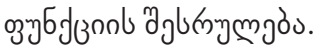

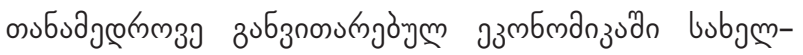

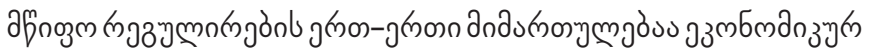

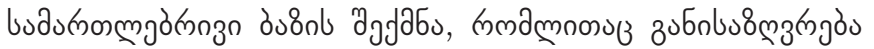

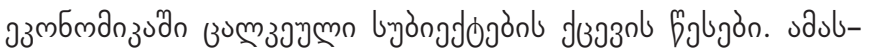

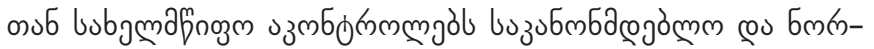

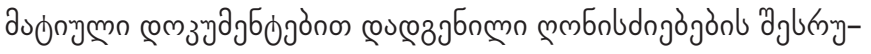
mjòbl.

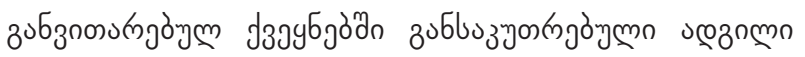

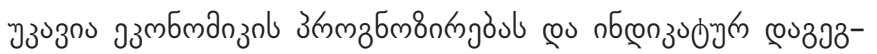

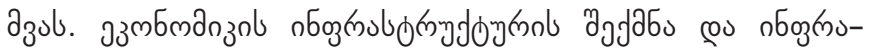

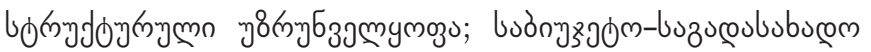

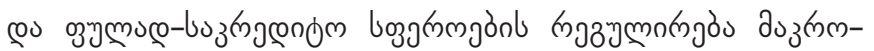

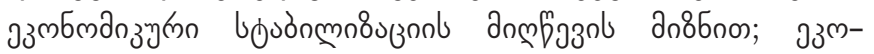

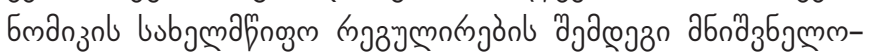

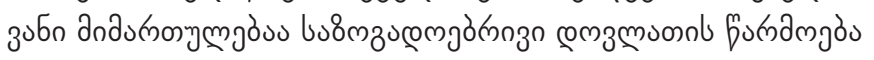

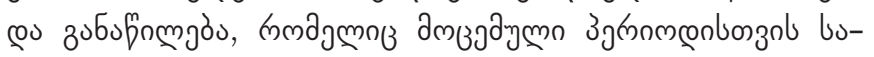

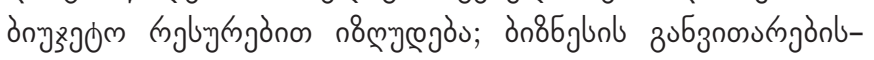

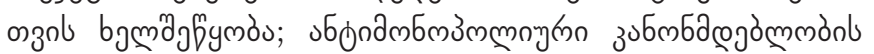

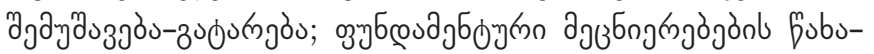

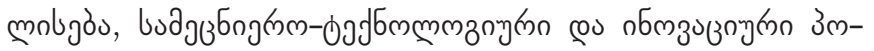

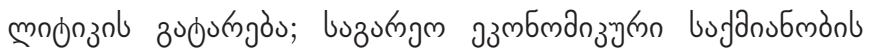

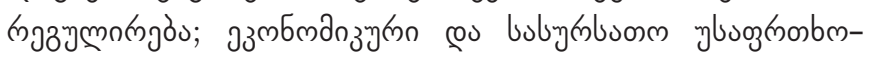

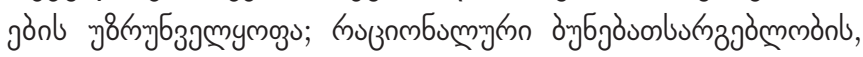

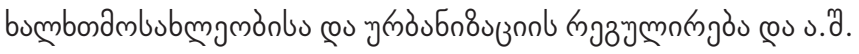

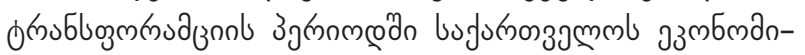

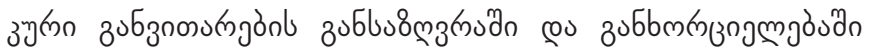

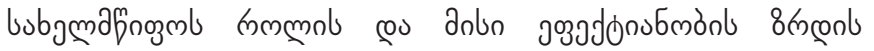

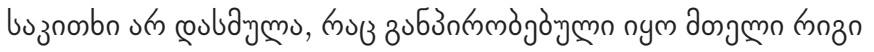
oुufom ringòno:

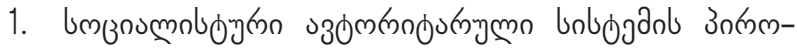

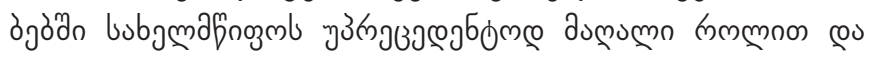

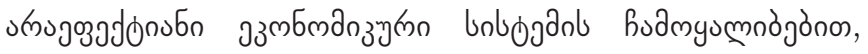

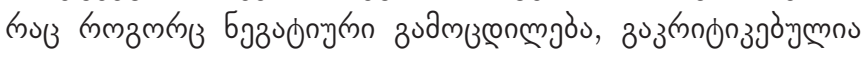

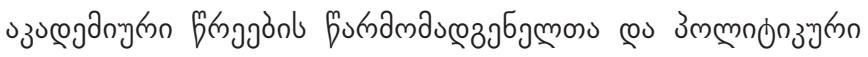
jmodol angr;

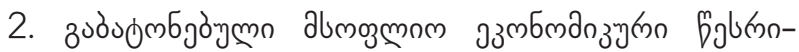

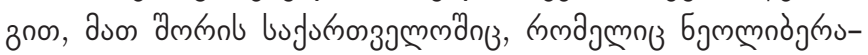

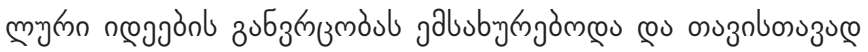

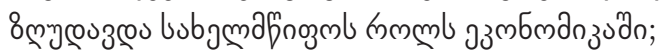

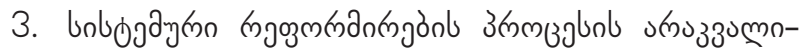

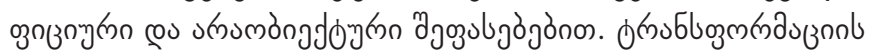

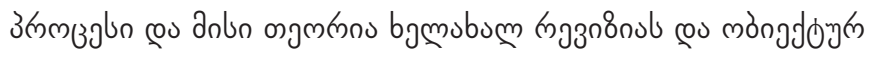
Ijogulbgoul amnosbmal;

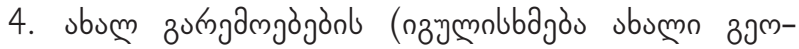

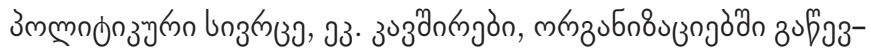

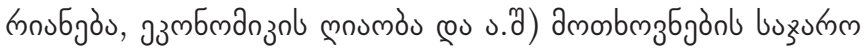

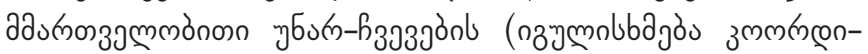

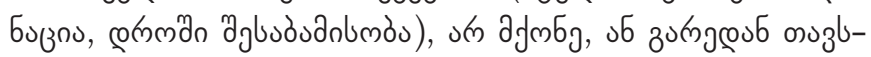

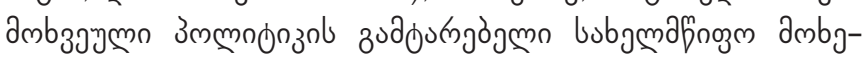

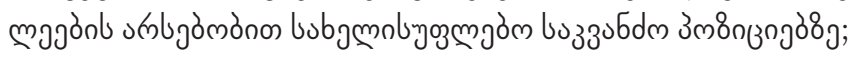

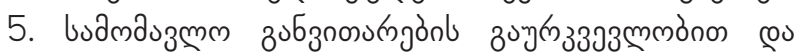

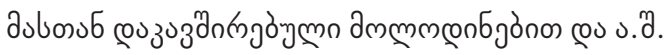

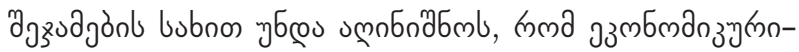

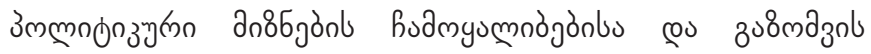

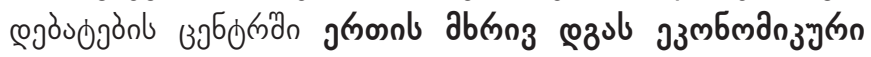

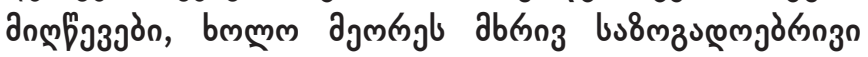

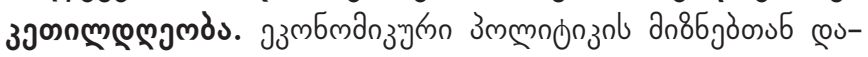
उ०3

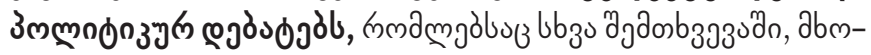

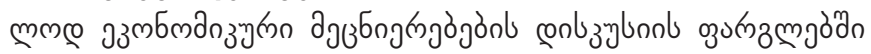

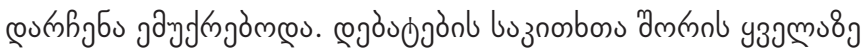

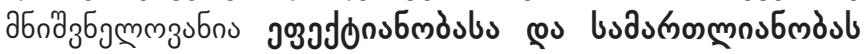

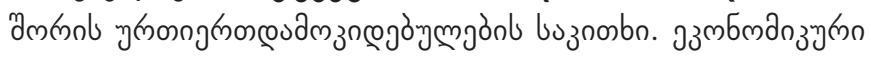

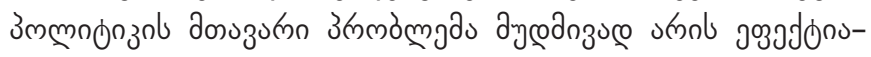

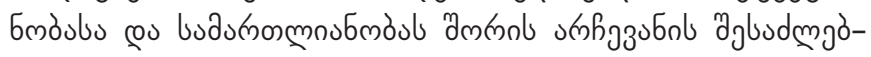

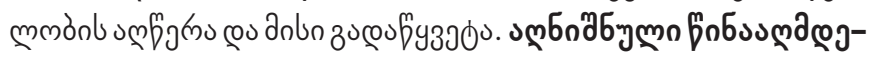

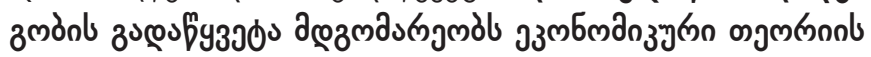

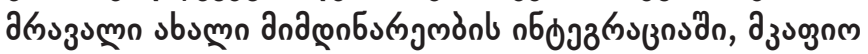

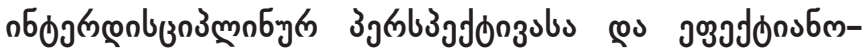

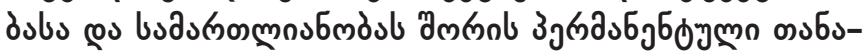

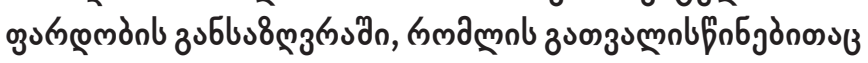

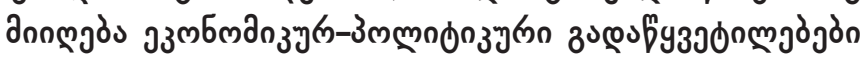

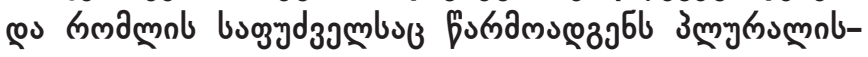

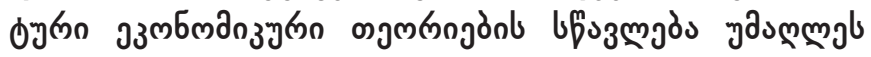

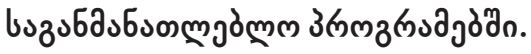

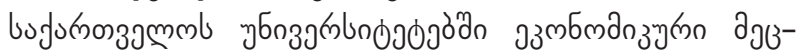

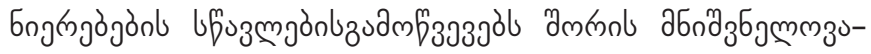

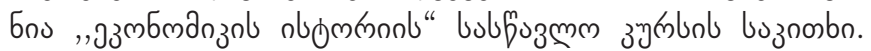




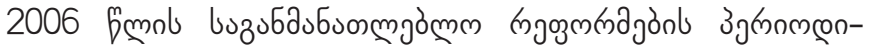

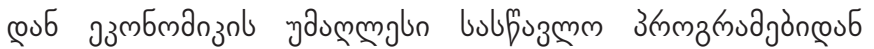

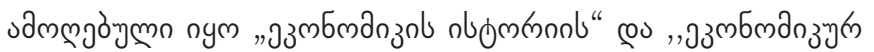
amd

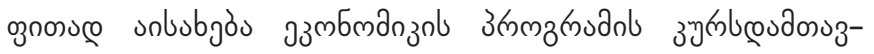

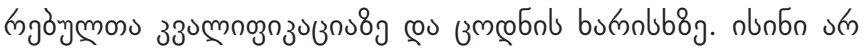

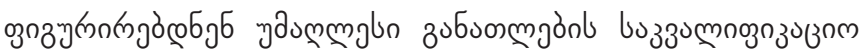

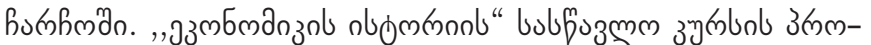

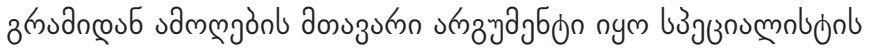

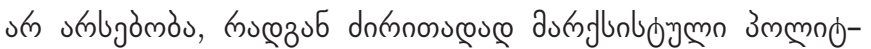

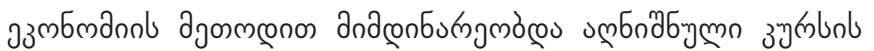

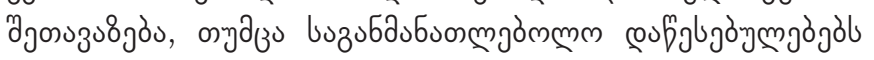

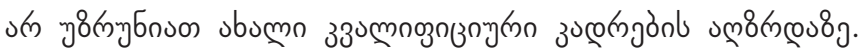

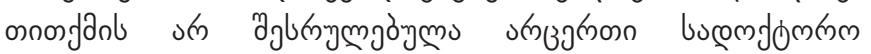

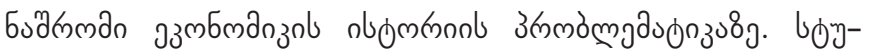

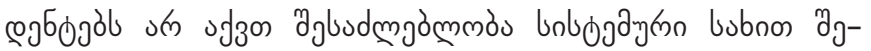

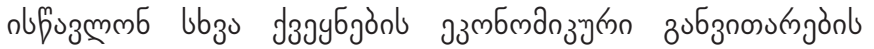

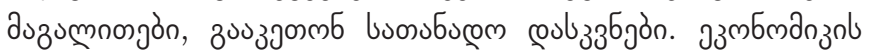

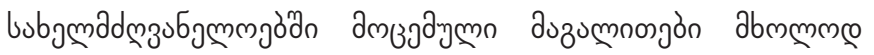

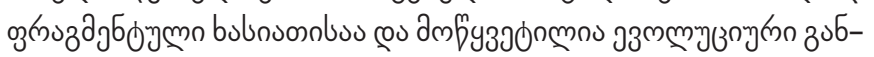

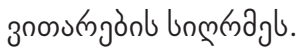

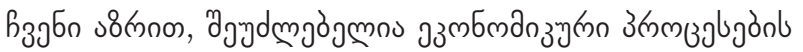

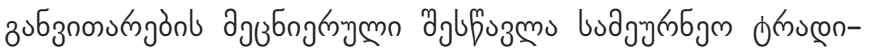

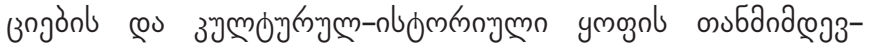

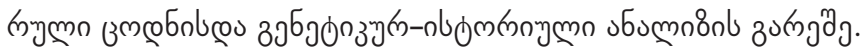

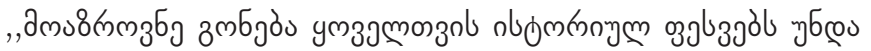

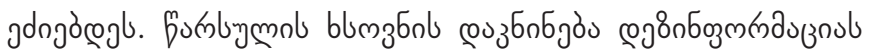

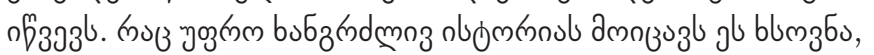

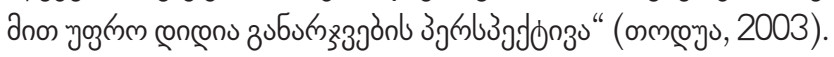

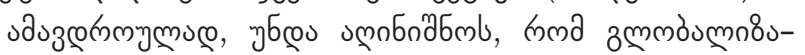

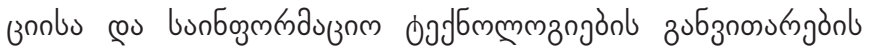

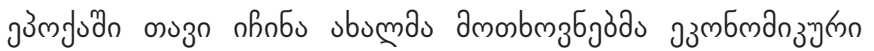

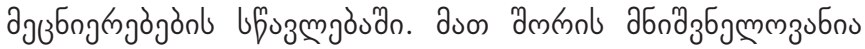

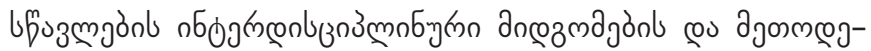

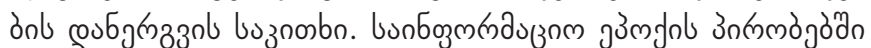

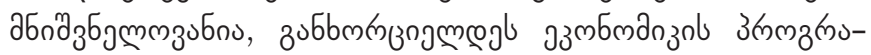

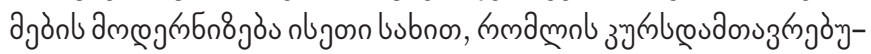

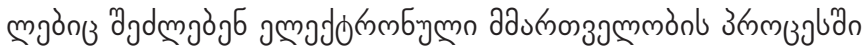

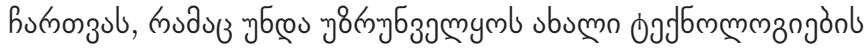

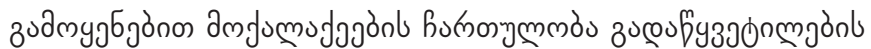

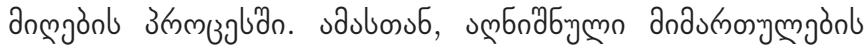

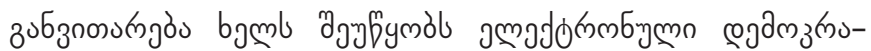

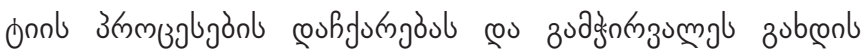

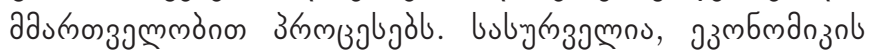

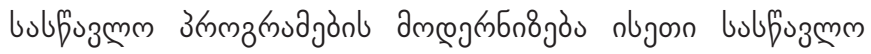

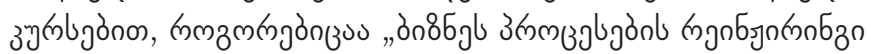

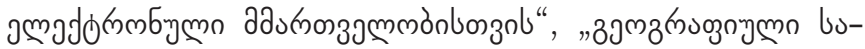

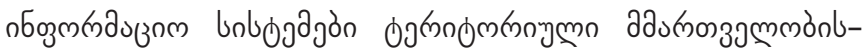

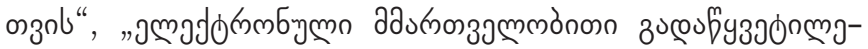

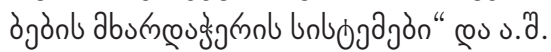

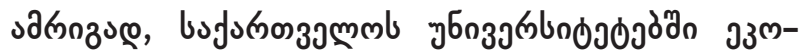

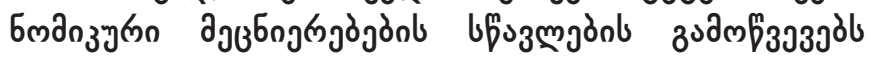

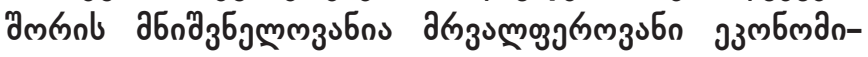

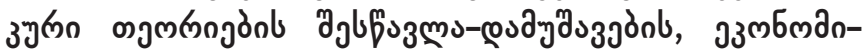
з

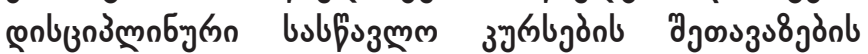

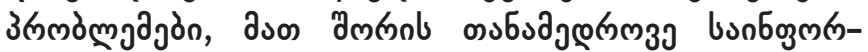

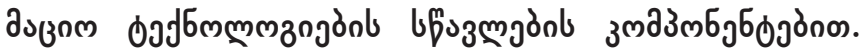

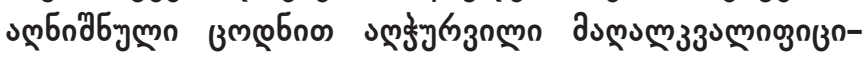

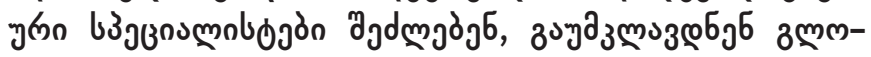

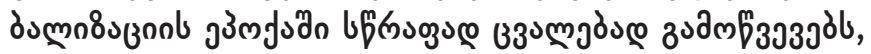

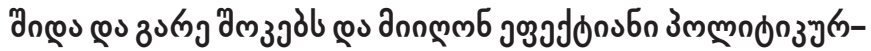

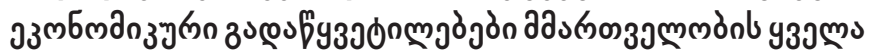

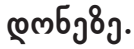

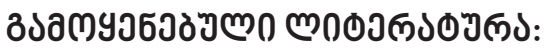

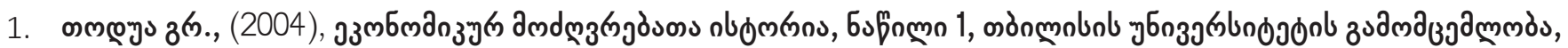
mònmoln, 622 33gmon;

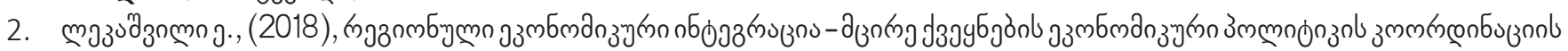

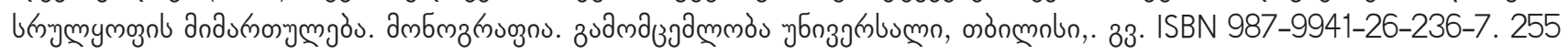

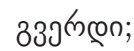

3. Gogorishvili, I.,(2016), Foreign economic policy of Georgia since gaining the Independence'. Estonian Discussions on Economic Policy, Topical issues in the EU Member States. 1/2016, Berlin-Tallin. ISSN 2228-1878,pp.33-47.http://www.mattimar.ee/publikatsioonid/majanduspoliitika/2016/1_2016_kroonika.pdf(Accessed 30 November 2017);

4. Gagnidze, I.,(2016), "The Impact of Entrepreneurial Universities on the Innovative Development of Economy", III International scientific and practical conference "Strategic Imperatives of Modern Management", KNEY, Kiev, pp. 186-192. http:// wiki.kneu.kiev.ua/bitstream/2010/20956/1/186-192.pdf (Accessed 11 December 2017);

5. Klump, R., (2015). Economic Policy. Instruments, aims and Institutions, third revised edition. Georgian translation. Publishing house of TSU,Tbilisi; 
6. Lekashvili, E., Gaprindashvili, G.,(2014). Socialist Integration in the Context of Regional Economic Integration(On the Example of the South Caucasian States),"Empires and Nations from the $18^{\text {th }}$ to the $20^{\text {th }}$ century", Volume 2, edited by AntonelloBiagini and Giovanna Motta. Cambridge Scholars Publishing. pp.142-152;

7. Lekashvili, E., (2011).Competition study under the conditions of globalization, Foregn Economic Relations Development in the Modern World Economic System, Intrnational Collaction of the Scientific Works, Kiev, Ostiva Ukrainy, pp.92-96;

8. Lekashvili, E.(2015), Entrepreneurial Way of Thinking and Its Development Challenges in Georgia. Journal L'Association 1901 'SEPIKE', Ed., 8, Poitiers (France), Frankfurt (Germany), Los Angeles (U.S.), pp. 121-126. ISSN 2196-9531. ISSN 23727438. http://docs.wixstatic.com/ugd/b199e2_004a4752ab114d47b94800998f727abb.pdf(Accessed 30 November 2017);

23. Lekashvili, E.,(2017), For The Study of development governmental strategy to support the entrepreneurial education in Georgia, THE 10TH ANNUAL EUROMED ACADEMY OF BUSINESS (EMAB) CONFERENCE Global and National Business Theories and Practice: Bridging the Past with the Future, Rome, (September 13-15, 2017), ISSN 2547-8516. pp.2107-2110; http://euromed2017.com/page/eka-lekashvili/(Accessed 7 February, 2018);

24. Lekashvili, E., (2018),Georgia: Communist Experiment and Transformational Economic Policy, Dynamics and Policies of Prejudice from the Eighteenth to the Twenty-first Century, Edited by Giovanna Motta, Cambridge Scholars Publishing, ISBN (10):1-5275-0862-5-3;ISBN (13): 978-1-5275-0862-0. pp. 395-411;http://lib.myilibrary.com/members_access.asp;http:// www.cambridgescholars.com/dynamics-and-policies-of-prejudice-from-the-eighteenth-to-the-twenty-first-century

9. Sepashvili, E., "Globalized World Economy, Innovations and National Policies for Economic Growth", Business Systems Laboratory $4^{\text {th }}$ International Symposium, 'Governing Business Systems. Theories and Challenges for Systems. Thinking in Practice', ISBN: 9788890824234, Vilnius, Lithuania, (2016), pp. 174-176.http://bslab-symposium.net/Vilnius.2016/BSLabVilnius2016-e-book_of_Abstracts.pdf(Accessed 9 February, 2018);

10. Papachashvili, N.Global Trade Order: The lessons from Doha Round, Book of Abstract, Business Systems Laboratory $4^{\text {th }}$ International Symposium, "Governing Business Systems. Theories and Challenges for Systems. Thinking in Practice", ISBN: 9788890824234, Vilnius, Lithuania, (2016). pp.84-86.http://bslab-symposium.net/Vilnius.2016/BSLab-Vilnius2016-ebook_of_Abstracts.pdf(Accessed 20 February 2018). 


\section{CHALLENGES OF TEACHING THE ECONOMIC SCIENCES IN GEORGIAN UNIVERSITIES}

\section{EKA LEKASHVILI}

https://doi.org/10.35945/gb.2018.06.012

Academic Doctor of Economics, Associate Professor

Ivane Javakhishvili Tbilisi State University, Georgia

eka.lekashvili@tsu.ge

\section{KEYWORDS: ECONOMIC SCIENCES, MODERNIZATION OF TEACHING PROGRAMS}

\section{SUMMARY}

The teaching process of economic sciences must serve to train the competitive staff that will be successful in the field of economic theory and practical economics.

Under the formation of market economy and economic transformation, the neo-liberal economic theory replaced the teaching of Marxist political economy in the university education space, for which a favorable environment for the occupation of the prevailing position in the country and the university was caused by many external and internal factors.

But, in fact, we have still taken a stand that promotes unilateral development of economic thinking and excludes the pluralism of scientific thinking and the development of critical thinking. In the modern conditions, the use of "laissez faire" doctrine without the realization of state regulation is an abstraction from reality".

Among the challenges of teaching the economic sciences in Georgian universities is the issue of the "Course of Economic History". From the higher education programs of the economy, the course of "history of economics" has been taken out, which in turn negatively affects the qualifications of the graduates of the economic program and the quality of knowledge. The Scientific study of economic processes cannot be done without consensual knowledge of traditional traditions and cultural-historical existence and genetic-historical analysis.

Also, in the era of globalization and information technology development, new challenges emerged in the challenges of teaching economic science. It is important to introduce interdisciplinary approaches and methods of teaching. In the context of the information period, it is important to modernize economic programs in the form of graduates, who will be able to engage in e-governance, which should provide citizens with the use of new technologies in their decision-making process. In addition, the development of this direction will facilitate acceleration of processes of electronic democracy and make governance processes transparent. It is desirable to modernize educational programs through such training courses as "reengineering business processes for e-governance", "geographical information systems for territorial governance", "e-governance solutions support systems", etc. 